\title{
Correction to: Practical and clinical utility of non-invasive vagus nerve stimulation (nVNS) for the acute treatment of migraine: a post hoc analysis of the randomized, sham-controlled, double-blind PRESTO trial
}

\author{
Licia Grazzi ${ }^{1,13^{*}}$, Cristina Tassorelli ${ }^{2,3}$, Marina de Tommaso ${ }^{4}$, Giulia Pierangeli ${ }^{5}$, Paolo Martelletti ${ }^{6}$, \\ Innocenzo Rainero ${ }^{7}$, Pierangelo Geppetti ${ }^{8}$, Anna Ambrosini ${ }^{9}$, Paola Sarchielli ${ }^{10}$, Eric Liebler ${ }^{11}$, Piero Barbanti ${ }^{12}$ and \\ on Behalf of the PRESTO Study Group
}

\section{Correction to: J Headache Pain (2018) 19:98} https://doi.org/10.1186/s10194-018-0928-1

Following publication of the original article [1], the authors notified us that a part of the Figure 3 legend was omitted during the proofing stage.

The original publication has been corrected. The incorrect and correct figure legends are presented below.

- Originally published legend for Figure 3:

$\geq 1$-Point Reduction in Pain Intensity at 30, 60, and 120 Minutes for (a) First Attack and (b) All Attacks

- Corrected Figure 3 legend:

$\geq 1$-Point Reduction in Pain Intensity at 30, 60, and 120 Minutes for (a) First Attack and (b) All Attacks. Models are adjusted for the patients' baseline pain score, use of preventive therapies, and presence of aura; data for number of patients are unadjusted numbers. Abbreviation: nVNS, non-invasive vagus nerve stimulation

\section{Author details}

${ }^{1}$ Neuroalgology Unit, Carlo Besta Neurological Institute and Foundation, Milan, Italy. ${ }^{2}$ Headache Science Centre, IRCCS C. Mondino Foundation, Pavia, Italy. ${ }^{3}$ Department of Brain and Behavioral Sciences, University of Pavia, Pavia, Italy. ${ }^{4}$ Neurophysiology and Pain Unit, University of Bari Aldo Moro, Bari, Italy. ${ }^{5}$ IRCCS Istituto delle Scienze Neurologiche di Bologna, Bologna, Italy. ${ }^{6}$ Department of Clinical and Molecular Medicine, Sapienza University, Rome, Italy. ${ }^{7}$ Department of Neuroscience, University of Turin, Turin, Italy.

${ }^{8}$ Headache Centre, University Hospital of Careggi, Florence, Italy. ${ }^{9}$ IRCCS

* Correspondence: licia.grazzi@istituto-besta.it

The original article can be found online at https://doi.org/10.1186/s10194018-0928-1

${ }^{1}$ Neuroalgology Unit, Carlo Besta Neurological Institute and Foundation, Milan, Italy

${ }^{13}$ Department of Fondazione IRCCS Istituto Neurologico C. Besta, U.O.

Neurologia III - Cefalee e Neuroalgologia, Via Celoria 11, 20133 Milan, Italy Full list of author information is available at the end of the article
Neuromed, Pozzilli (IS), Italy. ${ }^{10}$ Neurologic Clinic, Santa Maria della Misericordia Hospital, Perugia, Italy. ${ }^{11}$ electroCore, Inc, Basking Ridge, NJ, USA.

${ }^{12}$ Headache and Pain Unit, IRCCS San Raffaele Pisana, Rome, Italy.

${ }^{13}$ Department of Fondazione IRCCS Istituto Neurologico C. Besta, U.O.

Neurologia III - Cefalee e Neuroalgologia, Via Celoria 11, 20133 Milan, Italy.

Received: 14 November 2018 Accepted: 14 November 2018 Published online: 07 January 2019

\section{Reference}

1. Grazzi et al (2018) Practical and clinical utility of non-invasive vagus nerve stimulation (nVNS) for the acute treatment of migraine: a post hoc analysis of the randomized, sham-controlled, double-blind PRESTO trial. J Headache Pain 19:98. https://doi.org/10.1186/s10194-018-0928-1 International License (http://creativecommons.org/licenses/by/4.0/), which permits unrestricted use, distribution, and reproduction in any medium, provided you give appropriate credit to the original author(s) and the source, provide a link to the Creative Commons license, and indicate if changes were made. 